\title{
Effects of geomagnetic disturbances on humans functional state in space flight
}

\author{
Tamara K. Breus ${ }^{1}$, Roman M. Baevskii ${ }^{2}$, Anna G. Chernikova ${ }^{2}$
}

\author{
${ }^{1}$ Space Research Institute Russian Academy of Sciences, Moscow, Russia \\ ${ }^{2}$ Institute of Biomedical Problems Russian Academy of Sciences, Moscow, Russia \\ Email: breus36@mail.ru
}

Received February $9^{\text {th }}, 2012$; revised March 21 $1^{\text {st }}, 2012$; accepted March $29^{\text {th }}, 2012$

\begin{abstract}
Investigations of the effect of geomagnetic activity influence on the heart rhythm regulation of cosmonauts during the expeditions onboard the Soyuz spacecraft, and the MIR orbital space stations was carried out for various durations of flight in weightlessness and, under control of groups of cosmonauts who were inspected under flight conditions outside the geomagnetic disturbances and in ground preflight conditions, during disturbances and without them. The four series of analytical researches in which the data about changes of characteristics of heart rate variability are demonstrated for the first time the presence of specific effect of geomagnetic disturbances on the system of vegetative regulation of blood circulation of cosmonauts during the flight. The response of cosmonauts' heart rhythm on the magnetic storm is depending on the initial functional background and, in particular, on the state of mechanisms of vegetative regulation (the duration of flight and adaptation to it).
\end{abstract}

Keywords: Heart Rhythm Regulation; Cosmonauts; Geomagnetic Activity

\section{INTRODUCTION}

Space weather hazards have been well-studied during the past few decades, mainly in connection with effects on technical systems. The biological effects of solar and geomagnetic activity have been largely ignored because the amplitudes of the electromagnetic fields (EMF) are small, typically one to several hundreds of nanoteslas. This is much weaker than electromagnetic noise of anthropogenic origin and about ten orders of magnitude less than the characteristic energies of biochemical reactions. During the past 20 years however more careful consideration has been given to possible no thermal mechanisms of interaction of biological systems with weak $(<1 \mathrm{mT})$ low frequency EMF, which are unavoidably present in the environment, and some progress has been made towards understanding how an interaction can occur.

Research results presented below were obtained in the late nineties the last century; however since then any new data concerning influence of geomagnetic disturbances on vegetative regulation of humans in conditions of space flight is not published. As this problem is still continue to be actual, especially in connection with interplanetary flights planned in the near future, authors have counted expedient to present the generalized review spent earlier studies with their modern treatment and taking into account a hypothesis about a role of rhythms of helio-geomagnetic activity in development of influence on adaptive reactions of biological systems.

\subsection{A New Hypothesis Concerning the Producing Biological Effects by Heliogeomagnetic Activity (Space Weather)}

It has recently been proposed [1-3] that heliogeomagnetic rhythmic variations may act as external synchronizers of biological rhythms in a manner similar to the effects of the variations of sunlight and temperature on diurnal (circadian) biorhythms due to the Earth rotation. The biorhythms of a biological system may be influenced by weak, regular fluctuations with noise, depending on the amplitude of noise, with the external regular signals being "trapped" and evolutionary integrated into its time structure when the system is in an unstable state-a phenomenon known as "stochastic resonance" or "stochastic filtration" [4]. Such a "magnetobiological" mechanism based on the results, obtained by Kirschvink et al. (1985) [19] on magnetosomes (iron components in some biological sells of humans and animals as receptors of electromagnetic signals) had been proposed in [5].

The impressive progress in this field, achieved in the recent years, is due to chronobiology - the science, that deals with the time organization of biological objects during their evolution and the external factors, winding the biological clock. The chronobiological studies were long focused on circadian (about one day) rhythms that 
formed in the course of evolution as a manifestation of the natural sunlight (temperature) rhythm, due to the Earth rotation. However, the recent studies have revealed the rhythms with periods of 3.5 days, about a week (7 days) about two weeks (14 days), and about 27 28 days, that significantly contribute to the endogenic rhythmic structure of all biological objects [6,7]. Figure 1(a) No external timers for these rhythms could be established. So, it was suggested that some of them did not arise as a result of natural evolution of biological systems, exposed to external effects, but were rather due to integration of the internal diurnal processes to organic properties of the system, completed for these particular time intervals. well as the associated geomagnetic field variations display the dominating period of about 27 days, corresponding to the solar rotation period and its harmonics and sub-harmonics, rhythms with periods of 3.5 days, about a week (7 days) about two weeks (14 days) (Figure 1(b)). There are also micropulsations of geomagnetic and electromagnetic fields (various type Pc -pulsations, and ionospheric Schumann resonances in the range of heart and brain rhythms [8-10].

\subsection{Previously Obtained Results}

The proposed hypothesis regards the rhythms of helio-

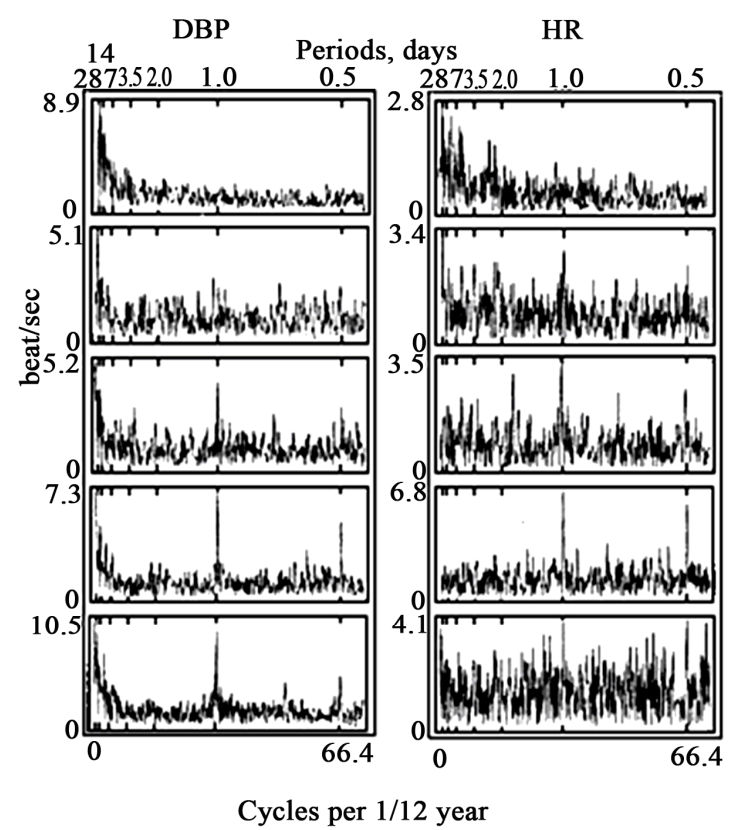

(a) geomagnetic activity, as well as the rhythm of the solar wave radiation, as a timer, playing the key role in selforganization of biological systems. We have carried out a number of studies that corroborated this concept. In particular, we found synchronous amplitude variations of the about weekly rhythm of biological and helio-geophysical characteristics for healthy people and new-born children both during a solar cycle, and during shorter time intervals. As follows from the proposed hypothesis, sharp variations in the rhythm of the timer (e.g. during geomagnetic storms) must be accompanied by stressreaction of biological objects, i.e. by adaptation.

However, as shown by space studies, the solar wind and interplanetary field characteristics, as desynchronosis, similar to the adaptation stress, disturbing the circadian rhythm during transcontinental flights. It is known that complex open non-linear systems are most sensitive to weak signal when they are in the state of internal instability. Therefore, it seemed reasonable to analyze the effect of geomagnetic variations on the "risk groups". There are several "groups of risk" depending on the nature of the instability of biological system, namely "undermined" (sick patients), "immature" (babies and small children) and "burdened by other stresses" (e.g. cosmonauts) [12].

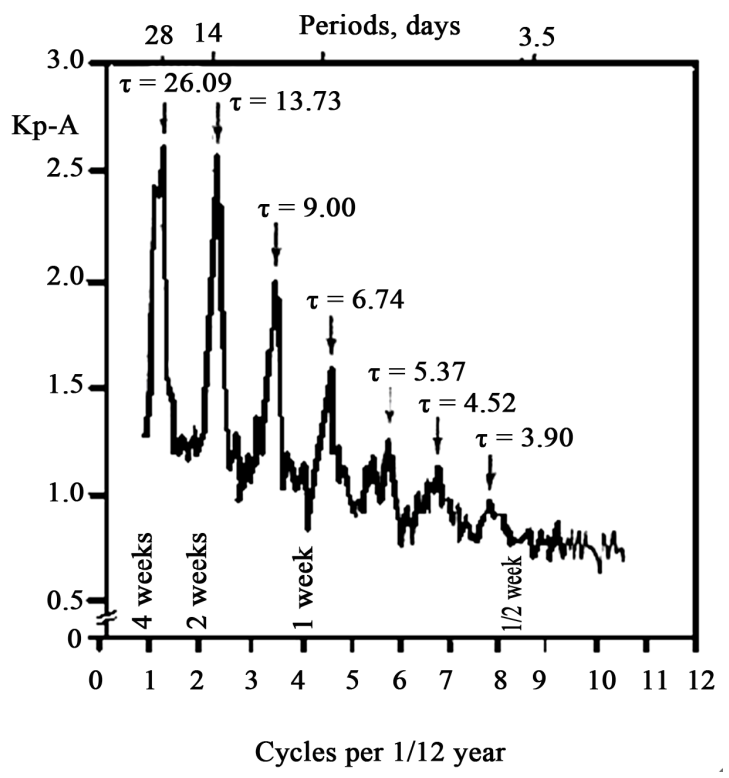

(b)

Figure 1. (a) Average spectra of heart rate (right) and diastolic blood pressure (left) based on 26 months monitoring of baby from his birth [11]. Amplitude of rhythm is shown along the ordinate axis (beats in min). The frequency (cycle/month) (down) and periods (upper axis) (in days). The period of monitoring from 26.01.89 to 19.03.91 divided by 5 corresponding series with four months in each. Obviously that there is a similarity of this spectra with the spectral components of Kp-index of geomagnetic activity; (b) Obtained for 38 years (1968-1995). The circadian rhythm ( 1 day) in baby rhythms induced by solar optical radiation, begin to appear only after 5 months, while periods similar to solar rotation and its harmonics, induced by solar corpuscular radiation and geomagnetic activity, exist already from the first month of life. 
Special studies to confirm suggested hypotheses using data obtained from laboratories and clinics had been performed in 1995-2003. These included statistical investigations of ambulance call data in Moscow (6 million ambulance calls and 10 different diseases); examinations of 172 patients in Moscow hospitals and 397 patients in Minnesota hospital (USA) $[1,13,14]$. Monitoring of the heart rate (HR) and blood pressure (BP) of 238 newborn babies was undertaken in Moscow and Minnesota (USA) [15]. There have been laboratory experiments and observations of animals (240 rabbits) in Moscow during magnetic storms in comparison with quiet geomagnetic conditions [16] (Figure 2).

Our laboratory observations of cardio-vascular characteristics of rabbits revealed the adaptation stress phenomena during a magnetic storm and the dynamics of the parameters, i.e. the heart rhythm stabilization and the loss of circadian structure during the main phase of the storm and a sharp decrease of the heart contractile power. This result was corroborated by examination of myocardial cells (cardio-myocytes) by electron microscopy that revealed their degradation and destruction in the main
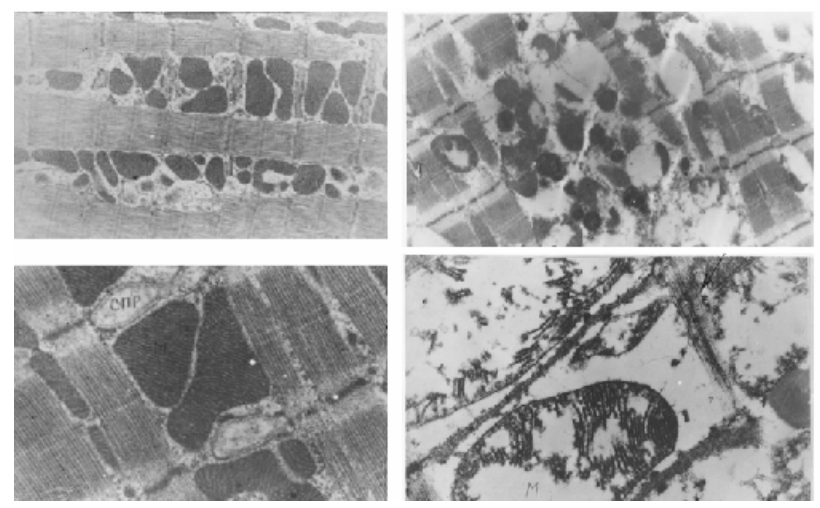

phase of the storm. In the recovery phase, the organism of animals also displayed a search for the optimal regime, when the rhythms were partly restored, but their amplitude was unstable and exceeded the normal value.

In clinical investigations some of the examined healthy volunteers of young and middle age in the ground-based group displayed a reaction to magnetic storm, accompanied by objective violations of the heart rhythm and variations of blood characteristics in microvessels. The stress caused by disease, increased the magnetic sensitivity of sick people. At the onset of geomagnetic storm, most of them manifested significant deviations in electrocardiogram (episodes of ST depression of rhythmdepending type), sudden jumps of arterial blood pressure, deceleration of blood flow, sludge formation and aggregation of erythrocytes, increased blood viscosity in arteries and in the places of decelerated blood flow. The storm-associated changes in the heart rhythm variability of sick people indicated to increased rigidity of the heart rhythm due to the changing balance of vegetative effects on the heart with the prevalence of the sympathetic system [17] (Figure 3).

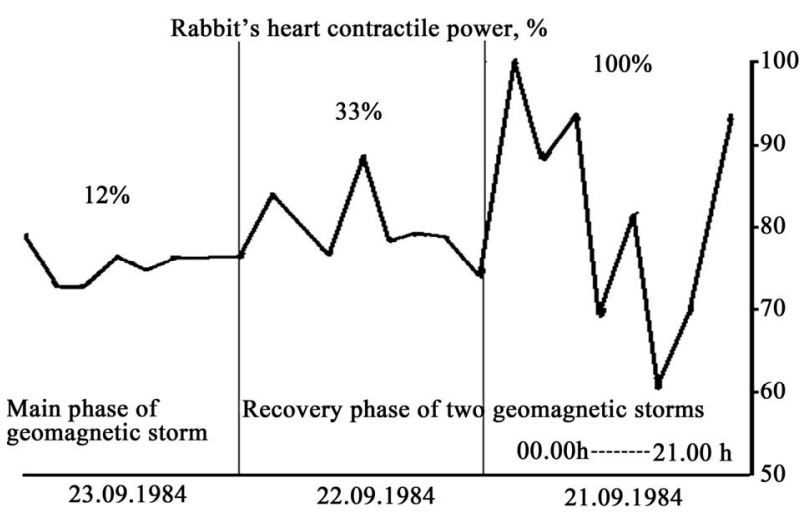

Figure 2. Normal structure of cardiomyocyte of a rabbit during quiet geomagnetic conditions (left) and cardiomyocyte of a rabbit during main phase of the magnetic storm (right) (the electronic microscopy with 20,000 enlargement for the same part of cardiac cell). Contractile power of rabbit's heart decreases during main phase of geomagnetic storms down to $12 \%$ in comparison with norm due to functional destruction mitochondria (compares left and right sites of photo) [16].
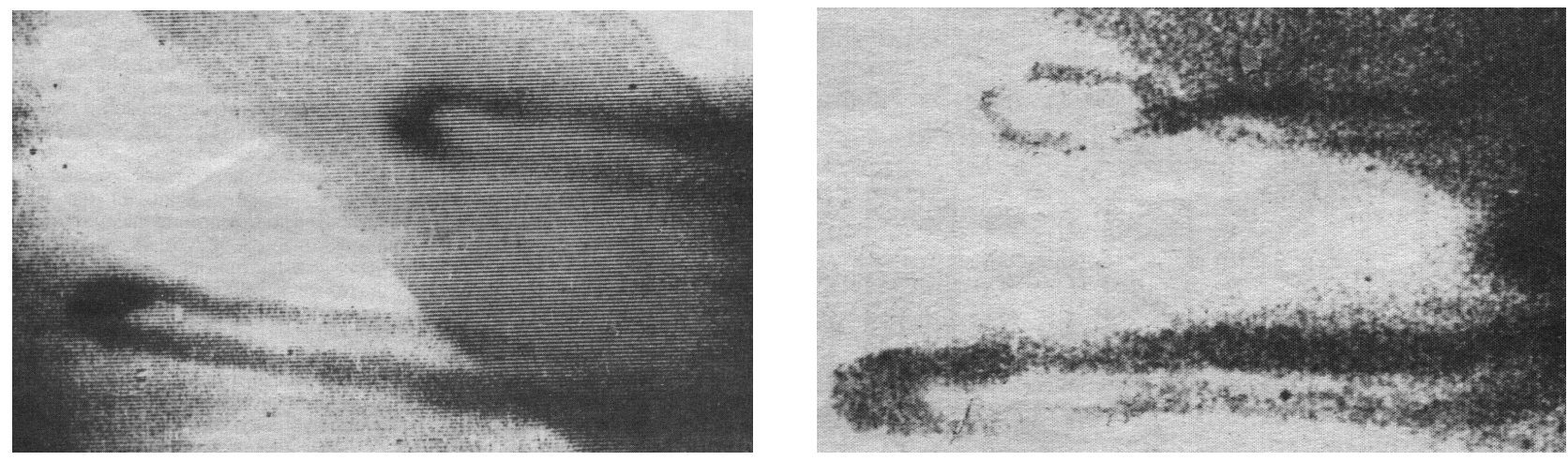

Figure 3. Nail capillary blood flow for patient with ischemic heart disease in quiet conditions (left) and after geomagnetic storm (right) (86 pts were investigated by [17]. Clear obvious erythrocytes coagulation and edema of capillary walls during the magnetic storm (right). 
To corroborate the character of the revealed reaction, we carried out a complex study of the magnetic storm influence on the production of steroid hormones and biogenic amines for the 26 patient with the ischemic heart disease and arterial hypertension and for 4 practically healthy people. The results showed a similar reaction of healthy and sick people to geomagnetic storm: violation of the production rate and ratio of gluccorticoids and mineral corticoids, i.e. a reliable increase of the hormone of adrenal glands (cortisone) in the diurnal urine. We also established a reliable increase of production of a number of biogenic amines, noradrenalin and dopamine in the same groups of patients, that corroborated the hypothesis stress reaction to the magnetic storm.

The production of melatonin (hormone of the pineal gland - epiphysis) in the human organism was studied for the first time during magnetic storms and under quiet geomagnetic conditions. It was shown that melatonin production of sick people during a magnetic storm was suppressed both in the day, and in the night-time [18]. The same result was reported in literature as obtained in the experiments with animals. Taking into account that the cortisol content in urine increases during a magnetic storm, it was interesting to see whether the adrenal glands, producing this hormone, might be "detectors" of geomagnetic field, the more so that they were shown to contain magnetite [19]. For this purpose, laboratory experiments were carried out to study the effect of the artificial ecologically significant variable magnetic field (VarMF) with a frequency of $8 \mathrm{~Hz}$ on animals with removed adrenal glands. It was shown that adrenalectomy modulates the development of general non-specific adaptation reactions of the organism (training, stress activation) as a response to VarMF, but does not change them in principle. Thus, it may be concluded that adrenal glands do not act directly as detectors of electromagnetic fields in the organism.

The early studies are clear evidence that the cardiovascular system is a main target for impact of geomagnetic disturbances $[1,7,11,13,15,20,21]$. However cardiovascular system is a target for effects of weightlessness in space flights too $[22,23]$. The disturbing factors here are some blood redistribution toward upper part of body and decrease of afferent flow from the periphery as well as decrease of energy budget. Baroreflex functions are decreasing while adaptation and compensation mechanisms that are operating in different autonomic centers have activated in a result of long-term flights. Thus both the long-term weightlessness and short-term geomagnetic disturbances affect the same functional systems of organism. Sensitivity to the negative effects of weightlessness has to be increased by this double impact and has to lead to deterioration of work capability and health state of cosmonauts.

\subsection{Objectives of This Paper}

According to above mentioned state of problem, cosmonauts, exposed to external stress of weightlessness, as well as people with decreased adaptation capacity due to a cardiovascular pathology can be included in "group of risk" undergoing of geomagnetic field disturbunces.

We realized studies carried out in the risk group of cosmonauts. Consequently we expected to observe violations of heart rhythm regulation in cosmonautes undergoing to exposure a magnetic storms: namely heart rythms variability stabilisation, increase or decrease heart rythm inself, a vegetative system violation and desynchronization typical for stress conditions. Experiments with cosmonauts seemed promising in the context of separation of effects from various potential affecting external agents, because the conditions for geomagnetic disturbance on the orbital man station are the same as on the Earth, whereas the typical accompanying factors, such as meteo-activity and infrasound, are eliminated.

Our analysis based on the mathematical analysis of heart rate data. This technique provides statistically comparable data on autonomic regulation of heart activity and have been used as in space flight medical care performances so in the ground conditions as a typical health care method in Cardiology [24]. There are wide archive data obtained in previous space expeditions that have been used partly here for our studies. There is also a large experience in clinical medicine and prophylactic care as well as in applied physiology as concern of use of proposed mathematical methods for the heart rate analysis $[25,26]$. These allow us to believe in significance of obtained new results never observed before.

\section{RESEARCH METHODS AND MATERIALS}

\subsection{Materials}

Four series of analytical examinations of diurnal records of RR-intervals of the heart rhythm of cosmonauts in space flights with various duration have been done here. The first analysis based on the archive records obtained on the initial stage of expeditions on the transport spacecraft SOYUZ for the period of 1990-1995. The records of 5 to 20 min duration which were obtained on the 32 nd circuit (the 1st day of flight) of the SOYUZ have been analyzed. The $32 \mathrm{nd}$ circuit is the last one before the SOYUZ docking to the MIR station and there is no special activity of cosmonauts at that orbit. The previous stresses obtained during start and transfer from extragravity to the weightlessness conditions are not affect more the crew on this circuit. The telemetry data of medical control of the heart rhythm are available just for this moment.

The 49 records (for 27 cosmonauts, 28 - 54 years old) 
have been selected. Eighteen cosmonauts completed their first flight, rest ones-2nd and 3rd flights. The three groups were constructed according to data on the geomagnetic activity, provided by the Institute of the Terrestrial Magnetism, Ionosphere and Radio Wave Propagation, Russian Academy of Sciences. There were 12 cosmonauts accomplished their flights during the geomagnetic storms over the period from 1990 to 1994 . Nine cosmonauts from whole expeditions had the 32nd circuit 1 - 2 day after the magnetic storm. The control group comprised 9 cosmonauts who had the 32 nd circuit in geomagnetic quiet conditions (separated more than 3 days from storms).

The second series of study have been devoted to the analysis of the diurnal Holter monitoring data for the two members of expedition N21 on the MIR space station obtained on March 21-23 1996 (on the 30th-32nd days after start of this flight). Scheme of proposed studies is shown in Figure 4(a). This particular period of space flight is characterized by activation and tension of regulatory mechanisms responsible for adaptation to the weightlessness. However stable adaptation is known to be starting only in a two-three months in weightlessness conditions. Thus the control group for comparison was carefully selected from the members of other expeditions (N6, N7 and N11) who accomplished their flights just in the same terms allocated not more than 4 - 5 days from selected for expedition N21 (Figure 4(a)).

The third series of study was devoted to the analysis of cosmonauts exposed to a magnetic storm at the end of

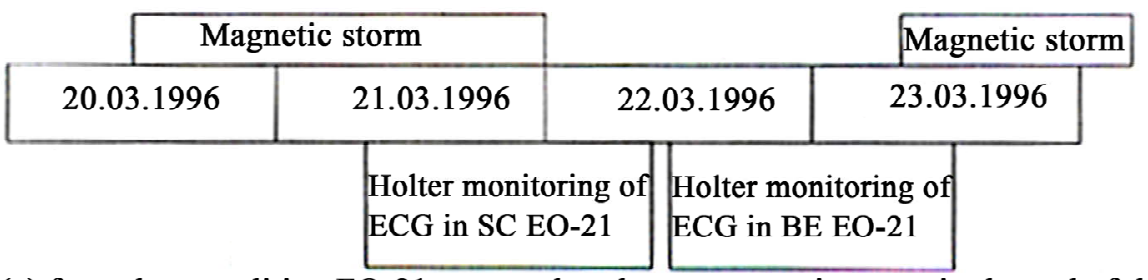

(a) from the cxpedition EO-21, cxposed to the geomagnetic storm in the end of the onc month flight

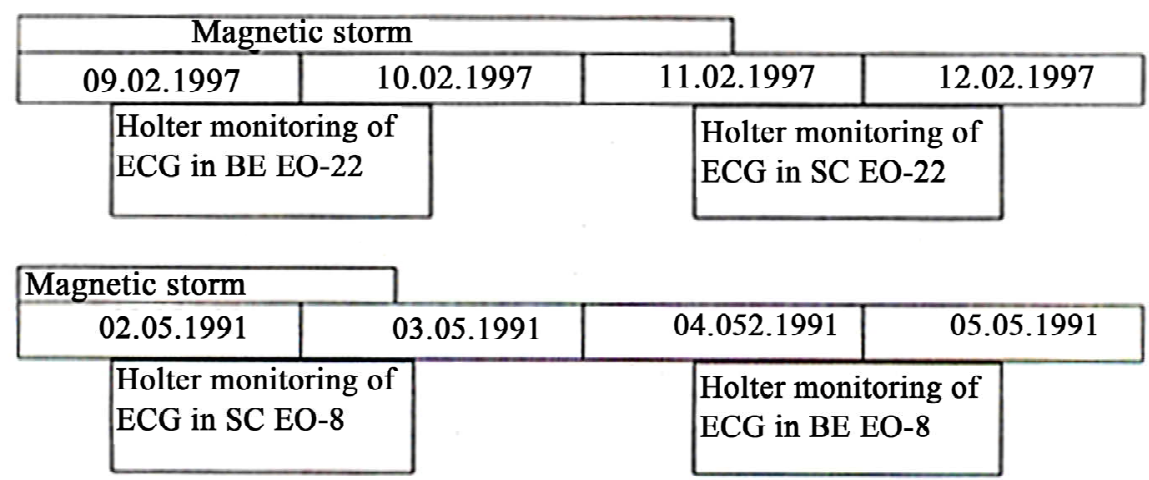

(b) from the expeditions EO- 8 and 22 in the end of the six month flight

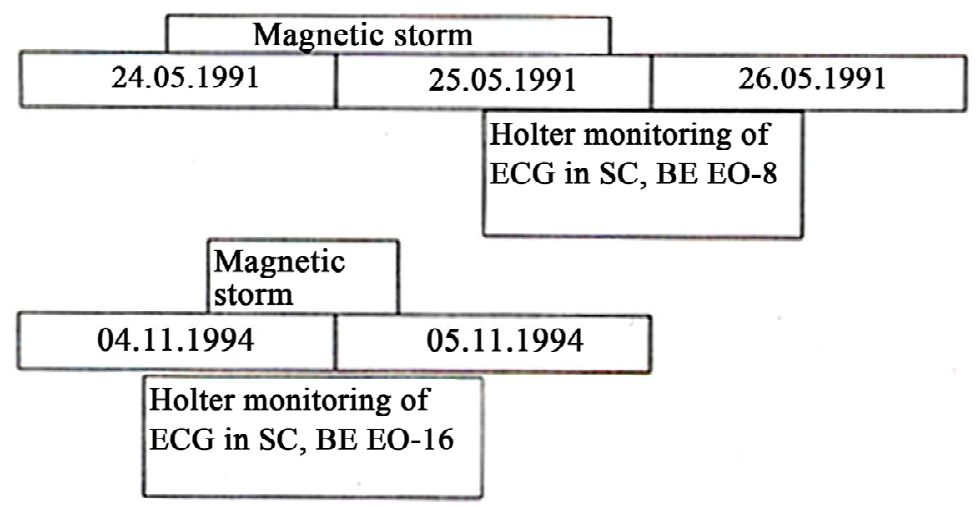

(c) from the expeditions EO-8 and EO-16 in day of landing

Figure 4. Shematic presentation of the scedule of the investigation of the autonomous regulation of the blood circulation in cosmonauts (the Holter monitoring data). 
six month of flight. The four Holter monitoring investigations of cosmonauts from expeditions N22 (February 9-12, 1997; 176th-179th days of flight) and N8 (May 2-5, 1991; 154th-155th days of flight) were analyzed. The scheme of experiments together with geomagnetic storm duration are presented in Figure 4(b). There were two cosmonauts from expeditions $\mathrm{N} 12$ and N17 in control group who have the same flight duration, but in the geomagnetic quiet conditions.

The fourth series related to the last stage of flight, namely, landing in the geomagnetic storm condition. The diurnal Holter monitoring records for the six cosmonauts in days of landings were analyzed. Three cosmonauts from expedition N8 and N16 comprised the group who landed during the geomagnetic storms. The scheme of experiments together with geomagnetic storm duration are presented in Figure 4(c). The three cosmonauts (from expeditions N11 and N15) belong to a control group. These data were analyzed very carefully because of low quality of usual data obtained during the first day of landing. Only the cleaned data were used for analysis.

\subsection{Methods}

A heart rate changes are the universal reaction of human organism to any external stress. However traditional average heart rate measurements characterize already established homeostatic mechanism, i.e. the final effect of many different regulatory impacts on the blood circulation. The main task of this mechanism is to provide balance between sympathetic and parasympathetic parts of autonomic nervous system (autonomic homeostasis). Different combinations of these two system branches activity sometimes can correspond to the same pulse rate. Besides the much more high level of regulation can affect the heart rate. These allow us to account the sinus node as a sensitive index for stress reactions in the process of adaptation to the environmental conditions.

The wide application of mathematical methods developed more that 30 years ago for the space cardiology are used now in order to reveal the reactions of cardiovascular system to be index of general adaptivity of organism [22] (Parin, Baevsky, Volkov, and Gazenko, 1967). The main information characterizing the heart regulation systems exists in the "scatter function" of cardiointerval duration.

It is well known now that there are several components of the heart rhythm: respiratory or sinus component, arrhythmia, slow and super slow waves of nonrespiratory origin with periods from $10 \mathrm{~s}$ to several tenth minutes. Non-respiratory sinus arrhythmia has heart rhythm oscillations with periods higher than $10 \mathrm{~s}$. Slow (nonrespiratory) waves are correlated with similar waves in arterial blood pressure. There are slow waves of 1st order, 2nd order as well as of higher order of values. The data analysis relies on the standard definitions of the HRV, recently published by the European Cardiology Society and North American Society on Electrophysiology [27].

Table 1 presents a brief physiological interpretation of the main of the 50 characteristics used in this work. In Table 2 the list of the basic parameters of variability of a heart rate according to the methodical recommendations made by group of the Russian experts is presented [28].

The heart rate variability has been analyzed using the Control software complex, developed by the Konto en-

Table 1. The main characteristics of the HRV and its physiological interpretation.

\begin{tabular}{|c|c|c|c|}
\hline $\mathrm{N}$ & Parameters & & Physiological interpretation \\
\hline 1 & HR & Heart rate & The integral level of the cardiovascular system activity \\
\hline 2 & SDNN & Standard deviation & $\begin{array}{l}\text { The integral effect of autonomic regulation of } \\
\text { cardcardiovascular system }\end{array}$ \\
\hline 3 & RMSSD & Root mean of sum of successive differences & Parasymphatetic part of autonomic system \\
\hline 4 & $\mathrm{HF}(\%)$ & $\begin{array}{l}\text { Power of high frequency spectral component in relation } \\
\text { to total power }\end{array}$ & The related activity of parasymphatetic system \\
\hline 5 & LF $(\%)$ & $\begin{array}{l}\text { Power of low frequency spectral component in relation } \\
\text { to total power }\end{array}$ & The related activity of vascular center \\
\hline 6 & VLF $(\%)$ & $\begin{array}{l}\text { Power of very low frequency spectral component in } \\
\text { relation to total power }\end{array}$ & The related activity of symphatetic system \\
\hline 7 & SI & Stress index & Level of regulatory system strain \\
\hline 8 & pNN50 & $\begin{array}{l}\text { Number of sucessive RR-intervals with differences more } \\
\text { than } 50 \mathrm{~ms} \text { (in } \% \text { to all } \mathrm{NN} \text { ) }\end{array}$ & The degree of parasymphatetic activity \\
\hline 9 & $1 \mathrm{C}$ & Index of centralization & The degree of autonomic control centralization \\
\hline 10 & IARS & Index of regulatory systems activity & The integral activity of regulation systems \\
\hline 11 & NArr & Number of arrhithmias & Level of arrhythmias \\
\hline 12 & $\mathrm{LFt}$ & Period of LF & The time of baroreflectoric regulation \\
\hline
\end{tabular}


Table 2. Analysis of HRV for crew members of the "Soyuz" transporting vehicle at the 32nd orbit of flight before, during and after the magnetic storm effect.

\begin{tabular}{cccc}
\hline $\begin{array}{c}\text { Heart rhythm variability } \\
\text { indicators }\end{array}$ & $\begin{array}{c}\text { Control group - without the } \\
\text { magnetic storm effect }\end{array}$ & $\begin{array}{c}\text { Group during magnetic } \\
\text { storm }\end{array}$ & $\begin{array}{c}\text { Group after the magnetic } \\
\text { storm effect }\end{array}$ \\
\hline HR & 65.941 & $62.860^{\#}$ & 66.786 \\
SDNN & 0.062 & $0.055^{\#}$ & 0.068 \\
RMSSD & 43.760 & $34.935^{*}$ & 36.862 \\
HF (\%) & 20.486 & $15.248^{*}$ & $10.620^{*}$ \\
LF (\%) & 47.388 & 49.582 & $40.925^{*}$ \\
VLF (\%) & 32.125 & 35.168 & $48.454^{\#}$ \\
SI & 66.829 & $81.599^{*}$ & 57.908 \\
PNN50 & 19.871 & $12.764^{\#}$ & 14.892 \\
IC & 4.488 & 6.677 & $9.411^{\#}$ \\
IARS & 0.921 & 1.545 & $2.029^{\#}$ \\
Narr & 1.157 & $0.272^{*}$ & $4.382^{\#}$ \\
LFt & 10.667 & $12.035^{*}$ & $14.396^{\#}$ \\
\hline
\end{tabular}

", Reliable difference from the control group $\left({ }^{*} p<0.05,{ }^{\#} p<0.01\right)$.

terprise (Moscow) in collaboration with the Institute of Biomedical Problems. The dynamic sets of cardiointervals were analyzed by methods that had specific features in each particular study. The records were represented as ASCII files, i.e. as columns of cardio intervals lengths in milliseconds. In the first series, the files were analyzed by discrete running method, when the samples with a standard length of $256 \mathrm{~s}$ at a step of $20 \mathrm{~s}$ were considered. The length of the ECG records in this series ranged from 6 to $20 \mathrm{~min}$, so that we had from 4 to 50 successive samples for every person.

The statistical significance of differences between the indices of the heart rhythm variability in different testing groups was determined by pair-to-pair comparison following the Student's t-criterion (CSS software) and by the method of stepwise discriminant analysis with mathematical simulation of the phenomena under Investigation (BMDP software). Analyzing the diurnal sets of cardiointervals, we divided the diurnal records into 5-min sections (300 s), each analyzed as a separate sample. The results were averaged over every hour, over every 8 hours (morning, evening, night), and over a full day using the special "Holter" program. The graphics were presented in result of these analyses (Figure 5).

\section{RESULTS}

\subsection{The Beginning Phase of Flight}

Based on the data of medical control of cosmonauts' cardiac rhythm changes at the 32 nd orbit of the flight were analyzed for all crews of the Soyuz transporting vehicles (TV) for the period of 1986-1995. This orbit was the last before Soyuz TV docking with the orbital MIR station, and it was the orbit, on which cosmonauts' organism functions have already been relatively normalized after their activation at previous stages of flight. In total, 49 records were selected for analysis (for 49 crew members including the members of both main and visitation crews). A group of 12 cosmonauts was selected, who were in flight during all days of geomagnetic disturbances for the analyzed period. In addition, two control groups were selected: 9 cosmonauts whose flight has fallen on the time 3 - 7 days apart from magnetic storms, and the second group included the remaining 32 cosmonauts [1].
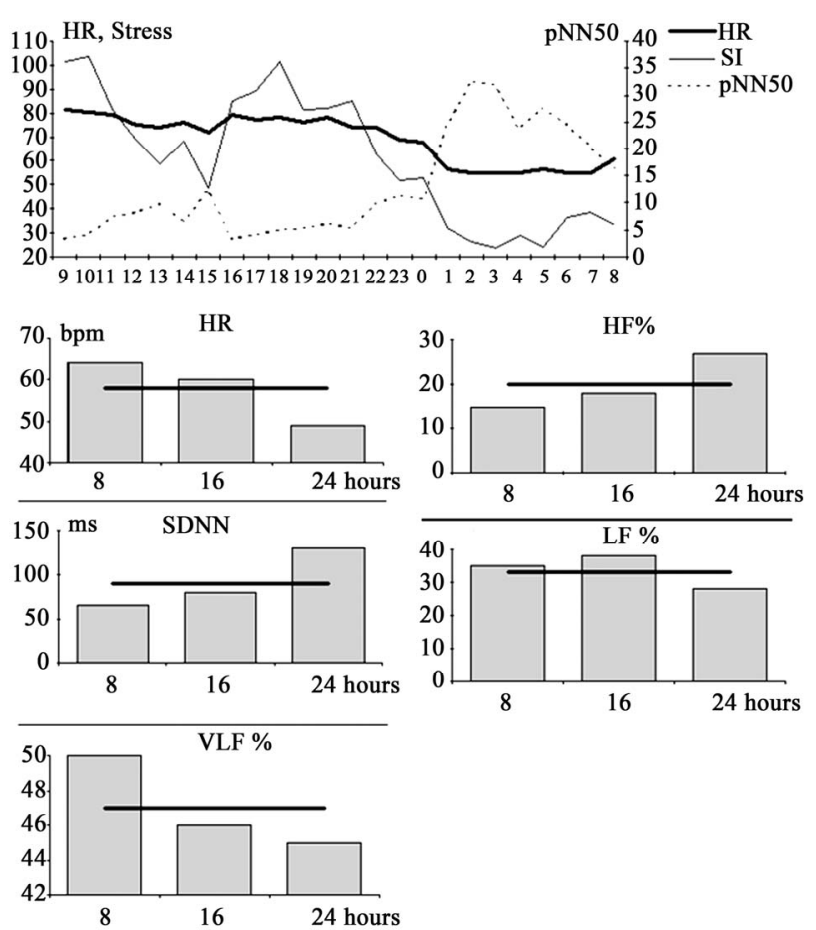

Figure 5. Examples of results of the analysis of the heart rate variability of the daily sets of cardiointervals. The upper panel shows the daily values of several parameters, lower panels show the averaged daily and 8-hours values some of them. 
Each record of a dynamic series of cardio intervals represented a text file of duration values of cardio intervals in milliseconds. The series length varied from 150 200 values $(2-3 \mathrm{~min})$ up to 1000 values $(15 \mathrm{~min})$. When analyzing the files the discrete-sliding method was used, where the samples with standard duration of $128 \mathrm{~s}$ were analyzed with a step of $10 \mathrm{~s}$. After this all calculated indicators were averaged. 40 various indicators were calculated by the techniques described above, a part of which is shown in Table 2 in the form of group means with estimation of the confidence of distinctions by the Student criterion.

The results show that the autonomic regulation processes differs significantly at the time of geomagnetic storm and 1 - 2 days after it was over. During the magnetic storm, the heart rate (Mo, HR) decreases with simultaneous shift of the autonomic balance towards the sympathetic regulation (decrease of $\mathrm{HF} \%, \mathrm{HFs}, \mathrm{CV}$, MxDMn, pNN50, RMSSD; increase of SI and VLFs). These implies the activation of the subcortical cardiovascular center. The increase of SNCA and LFs/HFs indices reveal specific variations in the vascular control, which is also corroborated by the growing of LFt index. These variations can be physiologically interpreted as activation of the vasomotor' center and deceleration of the reception and digesting of information in the latter. Thus the central nervous system and nervous regulation of vascular tonus are the targets for the geomagnetic field disturbances.

Some publications show that significant deterioration of following physiologic functions in seek people were observed $24-28 \mathrm{~h}$ after geomagnetic storm: increase of arterial blood pressure and crises of vegetative distonia $[20,29]$.

In our investigations, there was also significant deterioration of heart rhythm $1-2$ days after geomagnetic storms. The increase of $1 \mathrm{C}, \mathrm{VLF} \%$, VLFs together with significant decrease of HF were observed. The IARS and number of arrhythmia (Narr) were increased significantly as well. The latter fact is especially alarm.

Physiological interpretation of the storm-associated variations and in particular of those revealed in the subsequent 24 - 48 hours, clearly displayed deviations that range beyond the physiological norm. Figure 6 shows examples of obtained results for each of three separated groups of cosmonauts. The graphics of variability of cardiontervals shows clear decrease and smooth for groups affected by geomagnetic storms ((a): (2) and (3)). It is obvious also that auto-correlation functions (b) are smooth and have much slow decrease in groups (2) and (3) against group (1). The 2 nd order slow waves have predominance in the spectral functions (c) of heart rate in these groups.

These means that the geomagnetic storm increases the activity of regulatory systems and shifts the autonomic balance towards increasing of sympathetic activity in cosmonauts who accomplished the 1st day of space flight during magnetic storm. These results are in good agreement with papers where high sensitivity of autonomic nervous system to the geomagnetic disturbances were discovered [20, 29-31].

\subsection{One-Month Flight-(Crew of the Expedition E021 at the MIR Space Station) (Second Series of Studies)}

The second series of experiments was devoted to the study of the magnetic storm effect on the crew members on the 30th-32nd days of flight. The data for both cosmonauts were compared with the control data, obtained separately in the morning, evening, and night hours to eliminate errors due to the diurnal variations of the parameters under investigation. The results of statistical analysis of the daily mean heart rhythm variability indices are given in Table $\mathbf{3}$ for the control group and separately for the spacecraft commander (SC), and the board engineer (BE) in expedition N21 at the MIR station. As seen from this Table, the magnetic storm leads to a reliable growth of the heart rate (HR), the period (LFt) and the relative power of vasomotor waves (LF\%), while the heart rate variability (RMSSD) and amplitude of spectral maximum for the respiratory waves are decreased during this period [32].
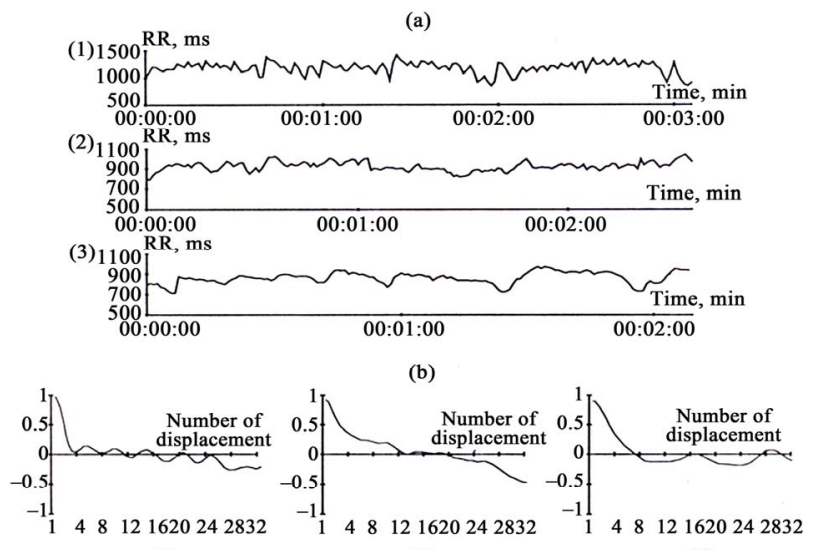

(1)

(2)

(3)

(c)

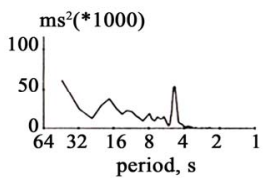

(1)

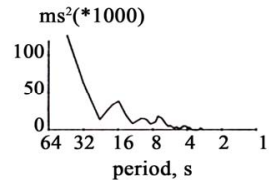

(2)

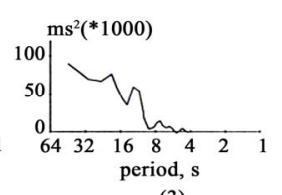

(3)
Figure 6. Examples of cardiointervalograms (a), auto-correlation function (b) and spectral power (c) obtained from data analysis of the heart rate variability of crew members (1) control group; (2) during magnetic storm; (3) 1 - 2 days after magnetic storms. 
Table 3. Daily mean indices of the heart rate variability in the control group and the space flight crew of the expedition E021 at the MIR space station exposed to the magnetic storm (30 32 days of flight).

\begin{tabular}{cccc}
\hline Parameters & $\begin{array}{c}\text { Control } \\
\text { group }\end{array}$ & $\begin{array}{c}\text { SC EO-21 during } \\
\text { magnetic storm }\end{array}$ & $\begin{array}{c}\text { BE EO-21 between two } \\
\text { magnetic storms }\end{array}$ \\
\hline HR & 61.361 & 65.757 & $69.053^{*}$ \\
SDNN & $0.076^{\prime}$ & $0.056^{\#}$ & 0.067 \\
RMSSD & 43.206 & $31.430^{*}$ & $38.221^{*}$ \\
HF (\%) & 13.818 & 13.919 & $10.640^{*}$ \\
LF (\%) & 41.283 & 42.349 & $46.525^{\#}$ \\
VLF (\%) & 44.898 & 43.730 & 42.834 \\
SI & 51.101 & $83.951^{*}$ & 60.294 \\
pNN50 & 19.333 & $8.242^{*}$ & 14.258 \\
1C & 9.857 & 9.887 & 10.669 \\
IARS & 2.591 & 1.738 & 2.331 \\
NArr & 4.288 & $3.937^{*}$ & $9.691^{*}$ \\
LFt' & 13.761 & $14.171^{*}$ & $14.046^{*}$ \\
\hline
\end{tabular}

${ }^{*},{ }^{*}$ Reliable difference from the control group $\left({ }^{*} p<0.05,{ }^{*} p<0.01\right)$.

The magnetic storm direct impact leads to the significant increase of stress index (SI) (sympathetic activation) and drop of the Regulatory Systems Activity Index (IARS) as well as to the most significant decrease of the heart rate variability (RMSSD). However, 24 - 36 hours after the geomagnetic storm the large heart rate (HR) increase and decrease of a relative power of respiratory waves (HF\%) have been observed. The decrease of power spectrum in whole frequency range with the same relative input of individual components is a very significant effect of the direct effect of the magnetic storm. It leads to activation of the highest levels of heart regulation systems, the highest autonomic centers. Such a shift of autonomic balance towards intensification of sympathetic nervous system reveals a nonspecific character of reactions and very high sensitivity of central nervous system to the geomagnetic perturbations. There are also specific reactions (some increase of the vasomotor wave's period (LFt). The more significant reactions of SC against of $\mathrm{BE}$ were connected with different time exposure of them to the magnetic storm. The heart rate variability variations in $\mathrm{BE}$, who have been exposed to the magnetic storm longer than $\mathrm{SC}$, shows some redistribution of power spectra over the frequency range: rise the $\mathrm{LF} \%$ and decrease of the $\mathrm{HF} \%$. There is quite significant increase the number of arrhythmia NArr (up to 9.69\%). These data corroborate the influence of geomagnetic variations on the vascular tonus and activation of the vasomotor center. The increased number of arrhythmias may be the evidence of the developing instability of the regulation mechanism due to "interference" of the highest-level blood circulation control into the autonomous regulation process.

Thus, the following conclusions are allowed to be done from the one-month flight data. The direct impact of the geomagnetic storm on the cosmonauts leads to specific reaction of vasomotor center as well as nonspecific activation of highest nervous centers of the heart regulation with shift of autonomic balance towards increase of the sympathetic nervous system tonus. The nonspecific variations of autonomic balance are typical for the period of relaxation after the magnetic storm. The large number of heart arrhythmias is typical for this period too.

\subsection{The Six-Month Flight}

Further, the results of daily continuous monitoring of the Holter electrocardiogram were studied for two crew members of the MIR EI-22 expedition on the 176th179th days of flight (after 6 months, on February 9-12, 1997) and N8 (May 2-5, 1991, 154th-155th days of flight). The age of cosmonauts N1 and N2 was 44 and 37 years, respectively. There were two cosmonauts from expeditions N12 and N17 in control group who have the same flight duration, but in the geomagnetic quiet conditions. As evidenced by the experience of numerous past expeditions of duration up to 6 months, the functional state of cosmonauts in this period of flight was characterized by occurrence of the stage of relatively steady adaptation.

Based on the Holter monitoring results, the time database was generated, which included the files of initial values of all cardio intervals for the day (70 - 100 thousand numerical values, approximately). For statistical processing, the data on 5-min intervals were used, which then were averaged over all days, over the periods of a day (day, evening, night), and over hour intervals. Figure 7 presents the diurnal dynamics of indicators of cardiac rhythm variability of cosmonauts during geomagnetic storms and under quiet conditions at the 6-th month of flight.

Attention should be paid to the changes of values of basic indicators in the control group (as compared to the one-month flight data). This is, first of all, a higher heartbeat rate and lowered cardiac rhythm variability, as well as redistribution of frequency components of the spectrum to the side of prevalence of slow second order waves and increase of IC. All this indicates to activation and stress of regulatory mechanisms of a cardiovascular system after half-year stay in weightlessness conditions.

Table 4 summarizes the data of the statistical analysis 
of the daily means of heart rhythm variability measured in a control group and in experimental groups after six months of space flight. A special attention should be given to the changes in the main indices in the control group (compared with the date of the one-month flight). First of all it is an increased heart rate (HR), reduced heart rate variability, a redistribution of spectral frequency components. Toward second-order slow waves, and a higher index of centralization. All this implies growing activation and strain of regulatory mechanisms of the cardiovascular system after six months of weightlessness.

Against this background, under the magnetic storm effect the HR becomes confidently lower, and the cardiac rhythm variability (pNN50, RMSSD) grows. In this case, the relative and absolute power of vasomotor waves (LF\%, LFs) confidently grows, and the slow wave components (VLFs) become stronger. The LFt indicator is confidently lower, which means the absence of the vascular center overstress. However, in general, the indicated changes suggest increased activity of regulatory systems (IARS).

After the magnetic storm the increased total power of spectrum is conserved in all ranges, and the number of arrhythmias (NArr) considerably grows. On the whole, it is seen from the presented results that after six-month stay under weightlessness conditions the cardiac rhythm regulation system of cosmonauts under geomagnetically quiet conditions is already in the state of functional stress because of the long flight itself. The magneti storm effect, as a new stressor, disturbs the vegetative balance, established to this time, and causes short-term

Table 4. Daily means of indices of the heart rate variability in control groups and in the groups exposed to magnetic storms (data of the six-month space flights).

\begin{tabular}{cccc}
\hline Parameters & $\begin{array}{c}\text { Control } \\
\text { group }\end{array}$ & $\begin{array}{c}\text { During magnetic } \\
\text { storm }\end{array}$ & $\begin{array}{c}\text { After magnetic } \\
\text { storm }\end{array}$ \\
\hline HR & 68.295 & $62.459^{\#}$ & 64.434 \\
SDNN & 0.053 & $0.075^{\#}$ & 0.066 \\
RMSSD & 27.134 & $40.749^{\#}$ & $35.976^{*}$ \\
HF (\%) & 12.409 & 10.460 & 13.552 \\
LF (\%) & 40.282 & $45.799^{*}$ & 39.620 \\
VLF (\%) & 47.308 & $43.740^{*}$ & $46.827^{*}$ \\
SI & 96.365 & $58.730^{\#}$ & $67.389^{\#}$ \\
pNN50 & 5.811 & $17.671^{*}$ & $11.525^{*}$ \\
$1 C$ & 12.012 & 12.130 & $8.883^{*}$ \\
IARS & 1.493 & $2.847^{*}$ & $1.976^{*}$ \\
NArr & 1.689 & 3.025 & $5.536^{*}$ \\
VLFs & 0.959 & $1.674^{*}$ & $1.362^{*}$ \\
LFt & 14.415 & $12.789^{*}$ & 14.090 \\
\hline
\end{tabular}

${ }^{*}, "$ Reliable difference from the control group $\left({ }^{*} p<0.05,{ }^{*} p<0.01\right)$. overstress of regulatory mechanisms. In this case, the parasympathetic link of regulation strengthens.

To get a better insight into the mechanisms of magnetic-storm impact on the autonomic regulation of blood circulation, we now consider the variations recorded in various periods of circadian variations (Figures 7 (a)(h)). In the first half of the day (Figure 7 B) a distinct decrease of the heart rate (HR, Figure 7(a) B) is observed accompanied by growing SDNN (Figure 7(b) B), RMSSD, pNN50 (Figure 7(d) B), MxDMn, and decreasing AMo and SI (Figure 7(c) B). This point to a shift of the autonomic balance toward a strengthening of parasympathetic regulation.

The reliable growth of the activity of the vasomotor center (LF\%, SNCA) is stated. Yet, along with this, the absolute spectrum power is growing over all its ranges. The sharp increase of IARS from 1.5 to 3.0 (Figure 7(e) B) implies a disbalance of the observed changes, their inadequacy. The aftereffect of geomagnetic disturbances is the maintenance of increased RMSSD, pNN50, HF\%, HFs, IARS values. No increases are recorded in the activity of the vasomotor center. Obvious is a sharp rise of the number of arrhythmias (from 2.3 to 7.0). In the afternoon (Figure 7 B) the group directly exposed to the magnetic storms maintains its low heart rate; high over-
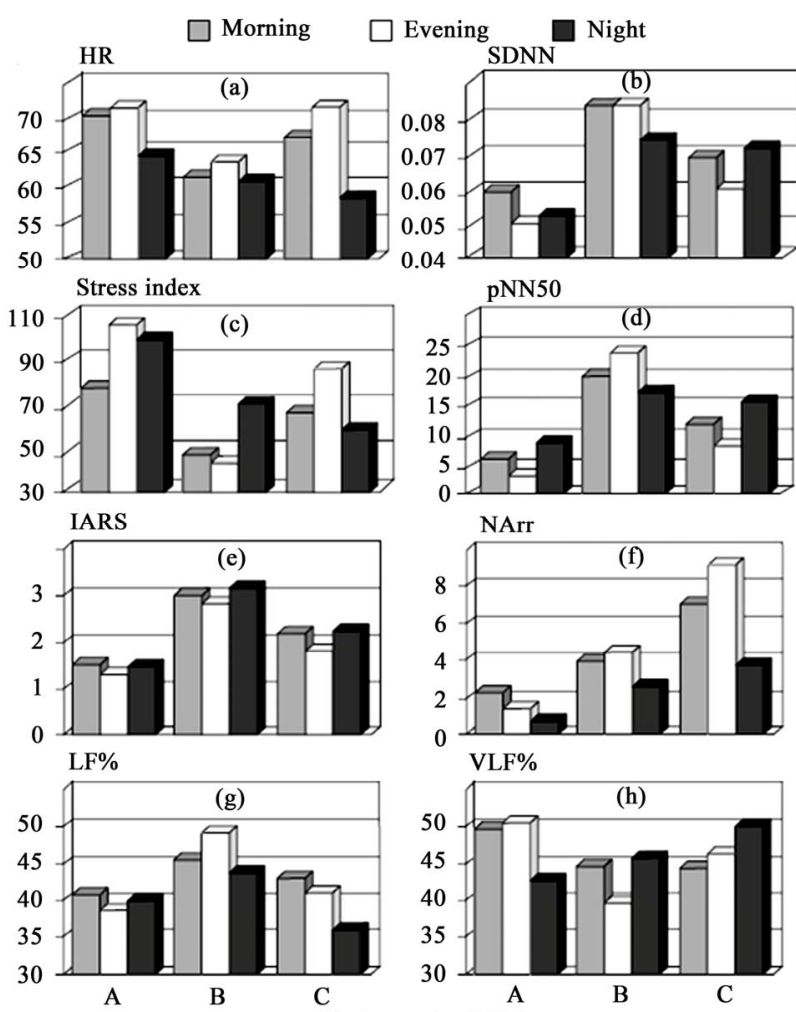

Sixth month of flight

Figure 7. Diurnal dynamics of cardiac rhythm variability indicators for cosmonauts of the control group (A) and groups during (B) and after (C) the effect of geomagnetic disturbances at the end of the 6th month of flight on the MIR station. 
all activity of regulatory systems (RMSSD, pNN50, SDNN, IARS) along with a. reduced activity of the sympathetic regulation, the activity of the vasomotor center grows $(\mathrm{LF} \%)$. It is important to mention the reliable reduction of LFt. The sum spectral power has reliably grown over all its ranges. The increased number of arrhythmias (Figure 7(f) B) is maintained. After the magnetic storm impact (Figure 7 C) the overall spectral power remains increased and the number of arrhythmias grows considerably.

During the nighttime and direct impact of the magnetic storm (Figure 7 B) a reliable increase of all rhythm variability parameters (SDNN, CV, MxDMn, RMSSD, pNN50) is observed as well as a decrease in the relative power of respiratory waves, an increase in the relative and the absolute power of vasomotor waves ( $\mathrm{LF} \%, \mathrm{LFs}$ ). Also growing is the index of centralization (1C). The absolute values of second-order slow waves (VLF) are reliably increased, as well as the number of arrhythmias. All these changes point to the presence of mainly specific changes of the vascular tonus, which are obviously closely associated with the activation of the highest autonomic centers. This is confirmed by the increase of spectral power in the range of second-order slow waves, which may be due to the down-going effects of higher cerebral structures. It should also be mentioned that the autonomic balance remains shifted toward parasympathetic activity amplification.

After the impact of a magnetic storm, all the above variations are mainly maintained, but the relative power of vasomotor waves is not increased. However a reliable increase in the relative power of second-order slow waves (VLF\%). This confirms that there exists a reaction of higher autonomic centers to the impact of a magnetic storm. As before, a reliable growth of the number of arrhythmias is maintained in this group.

\subsection{The Magnetic Storm Effects under Conditions of Landing (Cosmonauts Return from Flights)}

The results of the studies conducted on the day of cosmonauts' return to the Earth (4th series of studies) are of a great scientific and applied interest. The matter is that these variations develop against the background of psycho-emotional stress and non-specific stress due to the back exposure to the Earth gravitation after a long weightlessness conditions.

Table 5 summarizes the data on the differences between the indices of the heart rhythm variations in the first 4 hours and the next four hours (4 to 8 hours) after landing. As follows from Table 5, in the first hours after landing manifestations of psycho-emotional and gravitational stress considerably prevail over the reactions to the magnetic storm. In 4 to 8 hours the difference has already become obvious between the reaction of two groups of cosmonauts. Mainly such difference manifests itself as a decrease of the relative intensity of respiratory waves in the control group; that could be treated as a shift in autonomic balance toward the growing of the sympathetic activity. At the same time the relative and absolute intensity of second-order slow waves has reliable grown in the cosmonauts who landed on the day of a magnetic storm.

Along with this a reliable decrease of 2 nd-order slow wave intensity was also observed which against the background of the reliable heart-rate growth, may be interpreted as obvious mismatching of regulatory mechanisms. In the first hours on the Earth this group of cosmonauts demonstrates a tendency of higher rather than lower heart rate.

Thus, in the group of cosmonauts, landed on the day of a magnetic storm, variations in the vascular tonus were observed in 4 to 8 hours after landing, against the background of signatures of well-developed mismatching of central and autonomous regulation mechanisms. In the same period the main feature of the control group was the growth of activity of the sympathetic department of the autonomic nervous system against the background of the reliable decrease of the heart rate.

These results are very important for planning of reabilitation cares after a long-duration space flight. As it is known, one the most significant aspects of the reaction to a gravitational stress after landing is connected with the orthostatic stability, in particular a baroreflector link of the autonomic blood-circulation regulation. If there are any variations in the mechanisms of the vascular tonus regulation under the magnetic storm condition it will lead to the more long duration as well as to the large efforts for the restoration processes to the normal orthostatic stability.

Landing in the day of a magnetic storm after long

Table 5. Statistical differences between the indices of the heart rate variability in first 4 hours and next 4 hours after landing.

\begin{tabular}{ccccc}
\hline Parameters & \multicolumn{2}{c}{ Group AMS } & \multicolumn{2}{c}{ Group MS } \\
\hline HR & $0-4 \mathrm{hr}$ & $4-8 \mathrm{hr}$ & $0-4 \mathrm{hr}$ & $4-8 \mathrm{hr}$ \\
SDNN & 56.96 & $89.15^{* *}$ & 92.07 & $95.71^{*}$ \\
HF (\%) & 10.22 & $7.29^{* *}$ & 9.27 & $11.70^{*}$ \\
LF (\%) & 51.03 & 48.01 & 56.39 & $51.14^{* *}$ \\
VLF (\%) & 38.74 & $43.14^{* *}$ & 34.31 & $37.12^{*}$ \\
SI & 158.15 & $141.56^{* *}$ & $125.81^{*}$ & $134.85^{*}$ \\
HFs & 0.13 & 0.14 & 0.14 & $0.17^{*}$ \\
\hline *, \# & & 61.68 & 53.44 & 58.49 \\
\hline
\end{tabular}

\footnotetext{
${ }^{*},{ }^{*}$ Reliable difference from the control group $\left({ }^{*} p<0.05,{ }^{*} p<0.01\right)$.
} 
space flights can be followed by a danger for the life and health of the crew in case an emergencies. When the stresses are accumulated during landing, any new stress factor may cause an unpredictable reaction qualitatively different from what might be expected. The data on the impact of a magnetic storm on the autonomic regulation in the 6-month flight have demonstrated a qualitatively new response of the human organism against background of the stress "accumulated" in a long flight.

During the landing a "return stress" (gravitational and psycho-emotional) is imposed on the stress "accumulated" over the time of flight. The additional stress due to a magnetic storm may result is in quite unfavorable consequences.

Thus both a specific impact of a magnetic storm onto the regulation of vascular tonus and a non-specific stressfactor-induced reaction to the magnetic storm, may produce a similarly unfavorable effect on the post-landing condition of the crew members. Hence, one of the most essential practical recommendations following from the results of the study, is that of planning the return of cosmonauts after a long flights back to the Earth on the days when no magnetic storms are expected.

\section{DISCUSSION}

\section{Comparison of the One and Six-Month Space Flight Data}

Now we shall consider the similarities and differences in the reaction to the magnetic storms impact between a one-month and a six-month flight in weightlessness conditions. Table 6 compares the daily mean values of main indices of the heart rhythm variability measured during the above mentioned space flight periods.
Comparison of the daily indices of the heart rate variability in control groups and the testing groups of cosmonauts obtained during one-month and six-month fligt.

It is worthwhile to begin the analysis with a compareson of control groups where changes in the autonomic regulation of the cardiac rhythm are caused by the cosmonaut exposure only to space flight factors.

It follows from the data summarized in Table 6 that after the six-month weightlessness the heart rate (HR) grows and the heart rhythm variability decreases compared with the first months control group data (reliable changes recorded in SDNN, CV, MxDMn, RMSSD, AMo, pNN50). The SI is growing significantly which, together with the growth of heart rhythm stability (decrease of SDNN), is the evidence of high sympathetic activity of the autonomic nervous system. Relative spectral powers in the three frequency ranges do not change, however the $1 \mathrm{C}$ index is reliable increased.

The reliable reduction of absolute values of all power spectral ranges is in a well agreement with the reduction of the heart rate variability statistical characteristics. However, an activation of the highest autonomic centers is possibly involved because 1.5- to 2-fold reduction of spectral power taking place simultaneously over all frequency bands. Thus the in-flight dynamics of indices of autonomic regulation of blood circulation might be described by the two following arguments:

- During a six-month space flight the autonomic balance shifts toward the growing activity of a sympathetic regulation;

- This autonomic balance shift may possible be caused by the activation of highest autonomic centers.

The most important fact provided by the data compareson is the opposite variations of the autonomic balance

Table 6. Comparison of data for one month and six months flights.

\begin{tabular}{|c|c|c|c|c|}
\hline \multirow{2}{*}{ Parameters } & \multicolumn{2}{|c|}{ One-month flight } & \multicolumn{2}{|l|}{ Six-month flight } \\
\hline & $\begin{array}{l}\text { Control group } \\
\text { without magnetic storm influences }\end{array}$ & $\begin{array}{l}\text { Group during } \\
\text { magnetic storm }\end{array}$ & $\begin{array}{l}\text { Control group } \\
\text { without magnetic storm influences }\end{array}$ & $\begin{array}{l}\text { Group during } \\
\text { magnetic storm }\end{array}$ \\
\hline HR & 61.361 & $65.757^{\#}$ & $68.295^{*}$ & $62.459^{\#}$ \\
\hline SDNN & 0.076 & $0.056^{\#}$ & $0.053^{*}$ & $0.075^{\#}$ \\
\hline RMSSD & 43.206 & $31.430^{\#}$ & $27.134^{*}$ & $40.749^{\#}$ \\
\hline HF (\%) & 13.818 & 13.919 & 12.409 & 10.460 \\
\hline LF (\%) & 41.283 & 42.349 & 40.282 & $45.799^{\#}$ \\
\hline VLF (\%) & 44.898 & 43.730 & 47.308 & $43.740^{\#}$ \\
\hline SI & 51.101 & $83.951^{\#}$ & $96.365^{*}$ & $58.730^{\#}$ \\
\hline pNN50 & 19.333 & $8.242^{\#}$ & $5.811^{*}$ & $17.671^{\#}$ \\
\hline $1 \mathrm{C}$ & 9.857 & 9.887 & $12.012^{*}$ & 12.130 \\
\hline IARS & 2.591 & $1.738^{\#}$ & $1.493^{*}$ & $2.847^{\#}$ \\
\hline NArr & 4.288 & 3.937 & $1.689^{*}$ & $3.025^{\#}$ \\
\hline $\mathrm{LFt}$ & 13.761 & 14.171 & 14.415 & $12.789^{\#}$ \\
\hline
\end{tabular}

\footnotetext{
", Reliable difference from the control group $\left({ }^{*} p<0.05,{ }^{\#} p<0.01\right)$.
} 
under the magnetic storm conditions during one-month and six-month flights. In the one-month flight the reliable increase of the heart rate and reduction of the heart rhythm variability point to the activation of the sympathetic department of the autonomic nervous system. In the 6-month flight an opposite reaction with the parasympathetic activation (low heart rate and increased heart rhythm variability) is observed. In the first case the SI is reliable growing, in the second it is decreasing as reliable. Under the magnetic storm in the one-month flight the power of spectral components is reliable decreasing over all ranges, and it is growing in the sixmonth flight.

Under the impact of a magnetic storm relative values of spectral components do not vary in the one-month flight, whereas in the 6-month flight LF\% reliable grows and VLF\% decreases. Along with this, LFs/HFs is reliable growing in the six-month flight. During magnetic storms the six-month flight also reliable demonstrates the LFt decrease and the increase of the number of arrhythmia.

All the above mentioned differences show that variations in the autonomic regulation of blood circulation under the magnetic storm conditions considerably depend on the initial background. After six months in weightlessness conditions a new functional system of autonomic homeostasis forms. This new level of autonomic homeostasis differs by the shift of autonomic balance toward stronger activity of the sympathetic regulation. In this period the regulatory system remains in the state of functional stress and the impact of a magnetic storm, acting as a new stress-factor, violates the autonomic balance stetted by that time and apparently brings about a shortlived overstrain of regulatory mechanisms. This is accompanied by destabilization of the autonomic homeostasis, with the parasympathetic regulation getting relatively stronger.

Results of the researches of long duration flights conditions spent in the beginning of 2000th years have shown that at various stresses influences in case of an exhaustion of functional reserves, represent a prenosological states transition in premorbid and even in pathological states [33]. The concept of prenosological diagnostic has had development within of space medicine and now it is more widely used in various areas of medicine and physiology [34].

Adaptive reaction of organism to influence of space flight factors includes tension of regulatory systems as the protective mechanism due to which the homeostasis of the basic systems of organism remains at long action of weightlessness [35]. Additional pressure of geomagnetic disturbances can become "a trigger", which will cause transition from prenosological into premorbod state and even in pathology. Thus, magnetic storms in the condi- tions of long space flight increase risk of development of pathology.

\section{CONCLUSIONS}

In this paper it is shown for the first time that geomagnetic disturbances have a specific influence on autonomic regulation of blood circulation in cosmonauts during the flight dependently of duration of weightlessness conditions. Magnetic storms change the regulation of the vascular tonus and affect the autonomic balance both during their direct action and during the post time in the nearest 24 hours.

Hence, variations of the indices characterizing vasomotor center activity may be considered specific. Variations of indices reflecting the conditions of the sympathetic and parasympathetic tonus are secondary ones and may be regarded as a non-specific. Along with these specific and nonspecific variations there are unified reactions of blood circulation regulation mechanisms to the impact of a magnetic storm.

At the initial stage of a long space flight (on the 2 nd day and at the end of the 1st month flight), the effect of magnetic storms reconstructs the mechanism of regulation of the vascular tonus and changes the activity of the subcortical cardio-vascular center. This results in the development of a general non-specific stress reaction, which is characterized by enhanced tonus of the sympathetic nervous system (increased heart rate, reduced spectrum power of respiratory waves, etc.) and by involvement of the higher levels of physiological control in regulation processes. The reconstruction of regulation processes may be followed by increase of instability of the sinus node of accompanied by increased occurrence of the heart rhythm arrhythmias.

At the end of six-month flight, the magnetic storm may cause an activation of the parasympathetic regulation as a result of destabilization of the previously established autonomic homeostasis. In this period, the autonomic regulation system is under functional strain due to long duration flight in weightlessness. Any additional stress associated with the magnetic storm is likely to lead to a short-term over-strain of the regulation mechanisms. Thus, the reaction to the magnetic storm depends on the initial functional background, in particular on the state of the autonomic regulation mechanisms.

The group of cosmonauts who landed on the day of a magnetic storm displayed variations in the tonus of vascular regulation 4 - 8 hours after landing, consisting of increased heart rate against the background of deviations in the mechanism of autonomic regulation. It implies the deceleration of re-adaptation processes, which is of great practical importance for planning the recovery procedures after a long space flight. 
The studies of the geomagnetic storm effects on the humans in "risk group" (in conditions of external stress, weightlessness) strongly support the suggested hypothesis that geomagnetic storm sharply disturbs the rhythm of the external synchronizer of biological rhythms and is therefore accompanied by an adaptation stress reaction of the organism, which is most pronounced in the case of unstable state of adaptation system.

The functional conditions arising in an organism at geomagnetic disturbances are connected with changes of vegetative balance and are among prenosological states. Prenosological states are the first stage of adaptive reactions when for balance maintenance between an organism and environment conditions additional functional reserves is required, and this reaction is realized by tension of regulatory systems.

\section{ACKNOWLEDGEMENTS}

Authors express special gratitude to employees of Institute for medicobiomedical problems of Russian Academy of Sciences and in particular to G. A. Nikulina who have performed the part of work on data gathering and processing.

Many thanks to Academician of RAS and RAMS Prof. Anatoly Grigoriev, who was initiator of these studies and supported them over all time their development. By his initiative in 1995-1998 this work was supported by NASA grant and during last several years by grant of Russian Academy of Sciences "Fundamental sciences to medicine".

\section{REFERENCES}

[1] Breus, T.K., Halberg, F. and Cornelissen, G. (1995a) Biological effects of solar activity. Biofizika, 40, 737.

[2] Breus, T., Cornelissen, G., Halberg, F. and Levitin, A.E. (1995b) Temporal associations of life with solar and geophysical activity. Annales Geophysicae, 13, 12111222, doi:10.1007/s00585-995-1211-8

[3] Halberg, F., Cornélissen, G., Regal, P., et al. (2004) Chronoastrobiology: Proposal, nine conferences, heliogeomagnetics, transyears, near-weeks, near-decades, phylogenetic and ontogenetic memories. Biomed Pharmacother, 58, 150-187. doi:10.1016/S0753-3322(04)80025-8

[4] Gammaitoni, L., et al. (1998) Stochastic resonance. Reviews of Modern Physics, 70, 223-282.

[5] Binhi, V.N. and Chernavskii, D.S. (2005) Stochastic dynamics of magnetosomes in cytoskeleton. Europhysics letters, 70, 850-856.

[6] Cornelissen, G., Halberg, F., Breus, et al. (1998) The possible origin of the biological week' inferred from the human heart rate variations over the solar activity cycle. Biophysics, 43, 666-669.

[7] Cornelissen, G., Halberg, F., Breus, T.K., Syutkina, E.V., Baevskii, R.M., Weydahl, A., Watanabe, Y., Otsuka, K., Siegelova, J., Fiser, B. and Bakken, E.E. (2002) Nonphotic solar associations of heart rate variability and myocardial infarction. Journal of Atmospheric and Solar-
Terrestrial Physics, 64, 707-728.

[8] Kleimenova, N.G., et al. (2007) Pc1 geomagnetic pulsations as a potential hazard of myocardial infarction. Journal of Atmospheric and Solar-Terrestrial Physics, 69, 1759-1764.

[9] Cherry, N. (2002) Schumann resonances, a plausible biophysical mechanism for the human health effects of Solar/Geomagnetic Activity. Natural Hazards, 26, 279331. doi:10.1023/A:1015637127504

[10] Mitsutake, G., Otsuka, K., Hayakawa, M., Sekiguchi, M., Corndlissen, G. and Halberg, F. (2005) Does Schumann resonance affect our blood pressure? Biomedicine \& Pharmacotherapy, 59, 10-14.

[11] Halberg, F., Breus, T.K., Cornelissen, G., Bingham, C., Hillman, D.C., Rigatuso, J., Delmore, P. and Bakken, E. (1991) International womb-to-tomb chronome initiative group: Chronobiology in space. University of Minnesota, Minneapolis.

[12] Breus, T.K., Baevskii, R.M., Chibisov, S.M., et al. (1998) Impact of geomagnetic activity factors on human organism under extreme conditions and comparison with data of laboratory observations. Biofizka, 43, 811.

[13] Breus, T.K., Ozheredov, V.A., Syutkina, E.V. and Rogoza, A.N. (2007) Some aspects of the biological effects of space weather. Journal of Atmospheric and Solar-Terrestrial Physics, 69, 211.

[14] Villoresi, G., Breus, T.K., Iucci, N., Dorman, L.I. and Rapoport, S.I. (1994) The influence of geophysical and social effects on the incidences of clinically important pathologies (Moscow 1979-1981). Physica Medica, 10, 79-91.

[15] Syutkina, E.V., Cornelissen, G., Grigoriev, A.E., Mitish, M.D., Turti, T., Yatsyk, G.V., Pimenov, K., Breus, T.K., Studenikin, M.Y., Siegelova, J., Fiser, B., Dusek, J., Johnson, D. and Halberg, F. (1997) Neonatal intensive care may consider associations of cardiovascular rhythms with local magnetic disturbance. Scripta Medica, 70, 217-226.

[16] Chibisov, S.M., Breus, T.K., Levitin, A.E. and Drogova, G.M. (1995) Biological effects of a global magnetic storm. Biofizika, 40, 959-968.

[17] Gurfinkel, Y.I., Lyubimov, V.V., Oraevskii, V.N, et al. (1995) Influence of geomagnetic disturbances on capillary blood flow of patients with ischemic cardiac disease. Biofizika, 40, 800.

[18] Rapoport, S.I., Bolshakova, T.D., Malinovskya, N.K. and Breus, T.K. (1995) Magnetic storms as stress factor. Biofizika, 43, 844-848.

[19] Kirschvink, J.L., Jones, D.S. and MacFadden, B.J. (1985) Magnetite biomineralization and magnetoreception in organisms. Plenum, New York, 1985.

[20] Oransky, I.E. and Tzarfis, P.G. (1989) Biorhythmology and chronotherapy. High School, Moscow, 159.

[21] Otsuka, K., Yamanaka, T., Cornelissen, G., Breus, T., Chibisov, S.M., Baevsky, R., Halberg, F., Siegelova, J. and Fiser, B. (2000) Altered chronome of heart rate variability during span of high magnetic activity. Scripta Medica (Brno). 73, 111-116. 
[22] Parin, V.V., Baevsky, R.M. and Gazenko, O.G. (1965) Heart and circulation under space conditions. Cor et Vasa, 7, 165-184.

[23] Grigoriev, A.I. and Egorov, A.D. (1991) Advances in space biology and medicine bonting. JAI Press Inc., London.

[24] Baevsky, R.M., Nikulina, G.A. and Tazetdinov, I.G. (1984) Mathematical analysis of cardiac rhythm for estimation of specific features of organism adaptation to the space flight conditions. Vestnik AMN SSSR, 4, 62.

[25] Gazenko, O.G., Baevsky, R.M. and Yegorov, A.D. (1988) Space exploration and preventive medicine. 39 Congress of the IAF-Bangalor, Bangalore, 8-15 October 1988, 6.

[26] Van Ravenswaaij-Arts, C.M. and Kollee, A.A., Hopman, J.C.W., et al. (1993) Heart rate variability. Annals of Internal Medicine, 1184, 436-447.

[27] (1996) European Cardiology Society and North American Society on Electrophysiology. Circulation, 93, 10431065.

[28] Baevsky, R.M., Ivanov, G.G., Chireikin, L.V., at al. (2001) Analysis of heart rate variability using various electro-Cardio-graphical systems. Vestnik of Arythmo- logy, 24, 69-85.

[29] Muzalevskaya, N.I. (1973) The characteristic of indignant geomagnetic field as irritant. Problems of Space Biology, 123-142.

[30] Plehanov, G.F. (1978) Destabilization of unbalanced processes as basis of general mechanism of biological effect of magnetic fields.

[31] Presman, A.S. (1979) Electromagnetic signalisation in living nature. Soviet Radio, Moscow.

[32] Breus, T.K., Baevskii, R.M., Funtova, I.I., Nikulina, G.A., Alexeev, E.V. and Chernikova, A.G. (2008) Effect of geomagnetic field disturbances on the adaptive stress reaction of cosmonauts. Cosmic Research, 46, 367-372. doi:10.1134/S0010952508040138

[33] Baevsky, R.M. and Berseneva, A.P. (2008) Introduction to prenosological diagnostic. SLOVO, Moscow.

[34] Grigoriev, A.I. and Baevsky, R.M. (2007) Health concept and space medicine. SLOVO, Moscow.

[35] Baevsky, R.M., Nikulina, G.A., Funtova, I.I. and Chernikova, A.G. (2001) Vegetative regulation of blood circulation. Orbital Station MIR, 2, 36-68. 